\title{
Remote Sensing For Building Energy Simulation Input - A Field Trial
}

\author{
P. Gorzalka ${ }^{1}$, J. Estevam Schmiedt ${ }^{1}$, D. Frommholz ${ }^{1}$, M. Linkiewicz ${ }^{1}$, \\ D. Patel ${ }^{1}$, S. Plattner ${ }^{1}$, C. Schorn ${ }^{2}$, J. Göttsche ${ }^{2}$, B. Hoffschmidt ${ }^{1}$ \\ ${ }^{1}$ German Aerospace Center (DLR), Jülich/Cologne/Berlin/Oberpfaffenhofen, Germany \\ ${ }^{2}$ Solar-Institut Jülich at University of Applied Sciences Aachen, Jülich, Germany
}

\begin{abstract}
Accurate data about the condition and thermal properties of existing buildings are desirable for simulation purposes in the preparation of energy efficiency retrofits. However, established measurement methods tend to be laborious, time-consuming and expensive. Modern remote sensing technologies may capture the energetic characteristics of buildings in less time and at lower cost.

This paper presents first results of a field trial with RGB and thermal infrared (TIR) imagery taken from an unmanned aerial vehicle (UAV) to derive the geometry and heat transfer coefficients (U-values) for a single-family home as an input for energetic simulations. The outcome is compared to conventional analysis methods regarding accuracy and effort. It is shown that the building envelope can be precisely reconstructed using photogrammetry. However, the obtained U-values expose significant deviations. Potential error sources and possible remedies are discussed.
\end{abstract}

\section{Introduction}

Improving the energy efficiency related to space heating of existing buildings is an important factor in reducing man-made greenhouse gas emissions. Corresponding refurbishment measures are often economically beneficial for both building owners and residents. Nevertheless, they are not carried out as extensively and appropriately as they could be, because i.a. many owners do not know about the savings that can be realized on their property (Zundel and Stieß (2011)).

As a first step towards retrofit recommendations, different tools are available to assess the energetic performance of existing buildings:

- Using tabulated values for buildings similar in e.g. age or use.

- Inverse modelling based on time series data of energy consumption, weather and occupancy.

- One-time measurements of input parameters for building energy simulation.

The application of tabulated values requires little ef- fort, but does not account for building-specific properties. Inverse modelling can characterize thermal building performance parameters accurately, but requires the installation of various sensors and a sufficiently long monitoring period.

Regarding one-time measurements, several widely used methods cover various physical parameters related to the thermal performance of a building:

- Terrestrial laser scanning provides a threedimensional point cloud of outside and inside surfaces of the building.

- With the blower door test, the air exchange rate is determined and possible leaks in the building hull are detected.

- The U-values (overall heat transfer coefficients) of the envelope components can be calculated if the wall composition, material properties and layer thicknesses are known. Otherwise, the heat flux method (HFM) provides local values.

- Thermal infrared (TIR) images enable a qualitative evaluation of the building envelope in order to detect poorly insulated components and possible thermal bridges.

These methods are well-known, widely recognized by the community and relatively accurate. However, measurement campaigns and manual data evaluation work are time-consuming and therefore pricey.

The work presented in this paper is part of an ongoing research project that intends to make remote sensing technologies applicable for energetic analyses of buildings (Estevam Schmiedt et al. (2017)). The goal is to develop a tool box of measurement and evaluation methods to determine the energetically relevant properties of existing building envelopes quickly and accurately. Thus, crucial information for planning refurbishments on different scales from single buildings to entire city districts may be obtained in short time. More specifically, this publication describes the current progress in developing new and optimizing conventional data acquisition methods. The tool box under development aims at delivering building energy simulation input by solely relying on contactless measurements, general information (age, type, etc.) 
about the building, and data that its residents can obtain with little effort. In a first step, geometry and U-values are assessed for the example of a single family house. The polygonal hull as well as semantic and physical information is derived from photogrammetry and quantitative infrared measurements based on images taken from unmanned aerial vehicles (UAVs). Also, conventional analysis methods are applied and compared to the presented approach regarding accuracy and effort. Finally, it is evaluated how remote sensing can contribute to the fast and accurate generation of simulation input for existing buildings.

This paper is organized as follows: The Methods section introduces the analyzed building as well as the conventional and remote sensing methods applied in the field trial. The Results section focuses on measurement accuracy, duration and effort. The results for the different methods are compared in the Discussion section which also comments on model generation and data accessibility. The final Conclusion includes an outlook to future research.

\section{Methods}

The field trial took place at a free-standing two-story single-family house with typical wall structures and a gabled roof. It is easily accessible by UAVs from all sides. As the house is unoccupied and will be dismantled, extensive sample taking and deconstruction of its construction elements was possible. The entire building excluding the basement and attic space was heated electrically during a measurement campaign in Spring 2018. Room temperatures were continuously monitored meanwhile.

\section{Conventional reference measurements}

Energy efficiency parameters of the building were assessed conventionally as a reference for the remote sensing methods. Initially, the building was digitally reconstructed based on a 3D point cloud from outdoor and indoor laser scans using Autodesk Revit and Recap. With the OpenStudio plugin for SketchUp, energetically relevant information was subsequently introduced into the resulting building model:

- U-values of walls, roofs, windows and floors/ceilings as well as density, conductivity and specific heat (where applicable) based on literature values for the materials used for the structure.

- Data on thermal bridges, estimated from qualitative infrared thermography (IRT).

- The air infiltration rate, determined by a blower door test.

- Global position and orientation, derived from aerial images available online.

- The solar absorptance of the envelope, based on measured wall surface temperatures.

- Calculated values for surface convection.

The heating phase of the measurement campaign was then simulated using the software EnergyPlus and the previously created building model. The necessary heating load was derived from electricity use. Weather data and temperatures of the sky and soil were taken from weather stations nearby. To account for the blower door test on April 12, the air infiltration values measured during its execution were introduced. During the last days of the trial, wind velocities increased. As the air infiltration rate was constant in the model, the heating load for this particular period was adjusted to compensate for the higher heat loss.

\section{Remote sensing approach}

The remote sensing approach consists in the derivation of a $3 \mathrm{D}$ point cloud of the building from UAV imagery, the generation of a polygonal model and the recognition of windows and stories. Furthermore, it comprises UAV-based quantitative infrared thermography and the related data processing workflow.

\section{Point cloud derivation from UAV imagery}

Since they can be positioned freely within the space around a building, UAVs provide a flexible platform for close-range image acquisition. The recorded bitmaps can be used for high-resolution photogrammetric building reconstruction. In order to create a $3 \mathrm{D}$ model of the reference building, more than 1000 GPS/INS-tagged and hence roughly oriented RGB images were captured by a standard digital single-lens reflex camera (18 megapixels) mounted on a UAV. A subset of 152 bitmaps with sufficient overlap (more than $60 \%$ ) was selected manually and processed with the commercial software Agisoft PhotoScan. In this tool, after automatically detecting and assigning tie points inside the images, their initial pose gets refined, resulting in an accurate estimate for the global camera position and rotation at the time of exposure. The 3D point cloud subsequently is computed through dense stereo matching. It consists of approximately 12 million vertices at a spatial resolution of less than $1 \mathrm{~cm}$. Figure 1 depicts the final orientations for the selected images around the reconstructed point cloud.

\section{Generation of a textured polygonal 3D model}

The approach of Frommholz et al. (2017) is used to derive a polygonal $3 \mathrm{D}$ model with semantic annotations for the building. In its first step, the point cloud gets converted into a digital surface model (DSM) that encodes the elevation of the scene above the xy plane as a greyscale image. Having the DSM, a digital terrain model (DTM) showing the natural terrain only is obtained with the pyramidal height refinement algorithm described by Mayer (2004).

Wall surfaces are found by projecting the $3 \mathrm{D}$ point cloud onto the xy plane subdivided by a regular grid and analyzing its spatial distribution and $\mathrm{z}$ histogram for façade pieces. The pieces found get approximated by line segments using RANSAC (Fischler and Bolles (1981)). Neighbor segments again undergo linear re- 


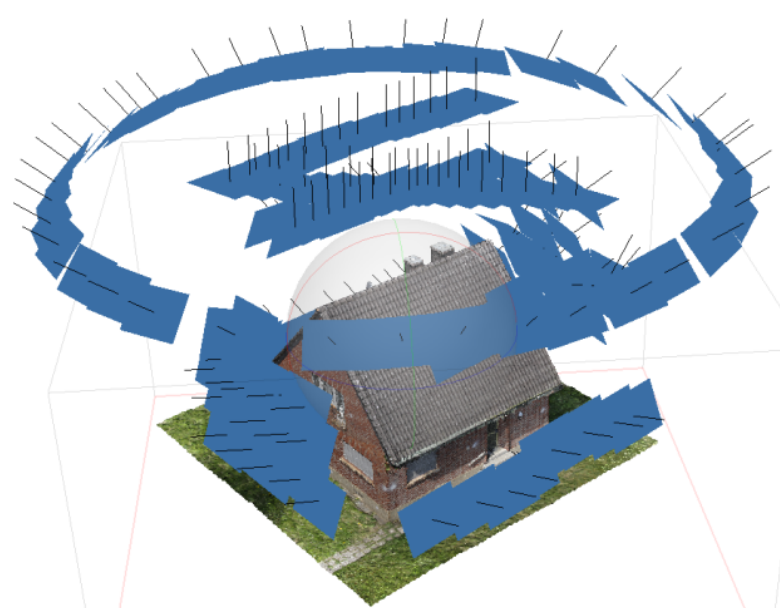

Figure 1: Camera positions around the 3D point cloud from Agisoft PhotoScan for the selected subset of nadir, oblique and nearly horizontal images.

gression when their directional difference is below a fixed angular threshold. The resulting façade fragments will be intersected if their end points are locally adjacent within the grid, forming the closed twodimensional contour of the building.

For the extraction of roof planes, slope and aspect of the DSM pixels within the area enclosed by the walls are computed, and similar pixels get grouped. During this process, chimneys and other installations on the top are filtered. Any gaps between or inside the roof segments are filled by breadth-first region growing yielding closed surfaces. The roof surfaces are then intersected with the walls which are orthogonally erected to match the terrain elevation. After adding the ground surface, the outcome of this process is a preliminary building hull with known surface semantics, i.e. roof, wall or ground labels.

Roof overhangs are extracted by analyzing the DSM outside of the area enclosed by the walls. The roof edges are virtually moved along their normal vectors with subpixel accuracy until a zero-crossing of the second-order derivative of the DSM in the gradient direction (representing the end of the overhang) is hit. The previously reconstructed roof polygons of the model then get extended accordingly.

For realistic renderings and for window recognition, any faces found so far are textured directly from the available input images. Each model face gets assigned a list of bitmaps sorted by their effective resolution on the respective 3D surface. Following a visibility test to bypass any occluders, unrectified polygonal pixel patches extracted from the remaining image candidates are written to a single texture atlas avoiding resampling whenever possible (see Figure 4). Perspective correction relative to the camera position is performed before both the atlas and the texture coordinates are added to the model. For further use, the model is stored as a CityGML file (OGC (2012)).

\section{Recognition of windows and stories}

To detect any windows inside the polygonal texture patches, the atlas bitmap is transformed into the huesaturation-value (HSV) color space that separates brightness from color. The histogram of the hue channel typically shows two significant peaks for windows and opaque façade parts. Since in most buildings the opaque area exceeds the window area, the minor peak comprising the lower histogram bins is considered to belong to the windows. The detected window pixel sets get filtered using a threshold for the minimum area and distance to the façade edges. The contours of the remaining patches are projected onto the wall polygons and added as window polygons to the CityGML representation of the building.

\section{Computation of floor area and heated volume}

Even without having any information about the indoor geometry, the detected window positions can be used to estimate the height of story-separating ceilings. For this purpose, the detected openings are classified as doors, ordinary windows or staircase windows. The lower edge of a door directly delivers the height of the floor of the story. When using an ordinary window, an approximate standard distance between the lower edge and the floor is considered.

From residents or building owners, information on which floors are heated and on the ceiling height of the basement can be obtained. With this data, the CityGML model is completed by adjusting the ground plate of the building, and both the heated volume and used floor area can be computed.

\section{Measurement of U-values using UAVs}

In recent publications, infrared thermography (IRT) has been tested to measure the U-value of building walls. Fokaides and Kalogirou (2011) and Tejedor et al. (2017) reached acceptable outcomes by recording images from inside a building. The measurement approach described by Patel et al. (2018) for external IRT with a camera on a UAV is used for the tool box presented in this paper and now being summarized. The U-value of an outer building wall $U_{\text {wall }}$ is equal to the heat per time and area passing through the wall in steady state, divided by the difference of indoor and outdoor air temperature.

$$
\begin{aligned}
U_{\text {wall }} & =\frac{\dot{q}}{T_{\text {in }, \text { air }}-T_{\text {out,air }}}=\frac{\dot{q}_{\text {rad }}+\dot{q}_{\text {conv }}}{T_{\text {in,air }}-T_{\text {out,air }}} \\
& =\frac{\varepsilon_{\mathrm{s}} \cdot \sigma \cdot\left(T_{\mathrm{s}}^{4}-T_{\mathrm{r}}^{4}\right)+h_{\mathrm{c}, \text { out }} \cdot\left(T_{\mathrm{s}}-T_{\text {out }, \text { air }}\right)}{T_{\text {in }, \text { air }}-T_{\text {out }, \text { air }}}
\end{aligned}
$$

The variable $\dot{q}$ stands for the specific heat flux through the wall. In steady state it is equal to the sum of the net specific heat fluxes emitted by the wall by radiation $\dot{q}_{\text {rad }}$ and by convection $\dot{q}_{\text {conv }} . T_{\text {in,air }}$ and $T_{\text {out,air }}$ are the air temperatures near the wall on the inside and on the outside respectively. In the second line of the equation, the radiative portion is expressed 
by the thermal emissivity of the outside wall surface $\varepsilon_{\mathrm{S}}$, the Stefan-Boltzmann constant $\sigma$, the average effective radiative temperature of the half space seen by the wall $T_{\mathrm{r}}$, and the surface temperature of the wall $T_{\mathrm{s}}$. For the convective part, $h_{\mathrm{c} \text {,out }}$ is the convective heat transfer coefficient on the outside of the wall. Instead of calculating the surface temperature, the radiation $W$ coming from the wall can be used to calculate the U-value. With the assumption that the surface behaves like an opaque grey body, the relation

$$
W=\varepsilon_{\mathrm{s}} \cdot \sigma \cdot T_{\mathrm{s}}^{4}+\left(1-\varepsilon_{\mathrm{s}}\right) \cdot \sigma \cdot T_{\mathrm{r}}^{4}
$$

can be used to replace $T_{\mathrm{s}}$ in (1) which gives

$$
\begin{aligned}
& U=\overbrace{\frac{W-\sigma \cdot T_{\mathrm{r}}^{4}}{T_{\text {in,air }}-T_{\text {out,air }}}}^{\text {radiative }} \\
& +\frac{\overbrace{h_{\mathrm{c}, \text { out }} \cdot\left(\sqrt[4]{\frac{W-\left(1-\varepsilon_{\mathrm{s}}\right) \cdot \sigma \cdot T_{\mathrm{r}}^{4}}{\varepsilon_{\mathrm{s}} \cdot \sigma}}-T_{\text {out,air }}\right)}^{\text {convective }}}{T_{\mathrm{in}, \text { air }}-T_{\text {out,air }}} .
\end{aligned}
$$

Patel et al. (2018) performed an uncertainty analysis based on this approach and measured values. Emissivity and indoor air temperature contribute less than one percent to the overall U-value uncertainty budget given a high temperature difference between inside and outside. Thus, the emissivity value can be taken from emissivity tables, and an inside air temperature sensor is not strictly necessary. To estimate the convective heat transfer coefficient, the model of Liu and Harris (2007) for the façade of a single-story building in a rural environment partially sheltered by tree belts and nearby buildings is suitable.

For the test building, TIR images were recorded in April 2018 in the early morning to minimize the influence of solar radiation and obtain a large air temperature difference between the outside and inside. The radiation coming from the wall surface was measured with a FLIR Duo Pro R (microbolometer detector) TIR camera. The reflected radiation was estimated from the radiation coming from crumpled aluminum foil attached to the wall. Table 1 lists all parameters used for U-value calculation and the corresponding sensors.

Table 1: Parameters required and sensors or sources used for measuring the U-value using IRT

\begin{tabular}{|c|c|}
\hline Parameter & Sensor/Source \\
\hline Emissivity & Reference table \\
Radiation from surface & TIR camera \\
Ambient refl. radiation & TIR camera \\
Outside air temp. & NiCr-Ni temp. sensor \\
Inside air temp. & NiCr-Ni temp. sensor \\
Wind velocity (for $h_{\mathrm{c}, \text { out }}$ ) & Vane anemometer \\
\hline
\end{tabular}

Furthermore, a HFM reference measurement of the U-value according to ISO 9869-1 (ISO (2014)) was carried out.

Due to possibly different surface materials, averaging radiation over the entire wall to get its $U$-value is unfavorable. There are two alternative approaches:

- Determining the U-value from a representative image region of the wall.

- Taking the weighted mean of the U-values derived for selected image areas of the façade.

Regarding the test building, the U-value was determined for a representative region close to the HFM reference measurement.

\section{Data processing}

Remote sensing data and additional information from different sources need to be merged for the energetic building simulation. Therefore, Python-based software to create an object-oriented digital building model was developed. Based on the semantically annotated envelope geometry from the CityGML file, objects for different building elements are created. During data import, small irregularities in surface planarity caused by the used reconstruction algorithm are compensated. Story-separating ceilings are introduced based on window positions, and the ground surface is moved downwards using the gathered information on the basement ceiling height. Measured U-values, as well as information on the thermal behavior of the windows and the heating system, both derived from literature (knowing their types), can be introduced as attributes.

Since the object notation in the software is based on CityGML, the format is the first option to exchange data with other applications. Its Energy application domain extension (Energy ADE) as described by Agugiaro et al. (2018) enables the introduction of thermal zones with dedicated boundaries and the attribution with energy-related parameters. Data stored in the format can also be directly visualized.

\section{Results}

As the goal of the project is to reduce the necessary amount of work for energetic building assessment, the presented results include insights regarding accuracy as well as time and effort to perform measurements and data processing.

\section{Validation of conventional measurements}

To validate the parameters of the building model generated from conventional measurements, the heating phase was simulated using EnergyPlus. Measured and simulated values of the mean building temperature throughout the campaign are shown in Figure 2. The measured room temperatures are mostly consistent with the simulation output. This even holds for the significant drop due to the blower door test on April 12. The cooling phase was not examined because of another blower door test immediately following the heating phase. Since the simulated tempera- 


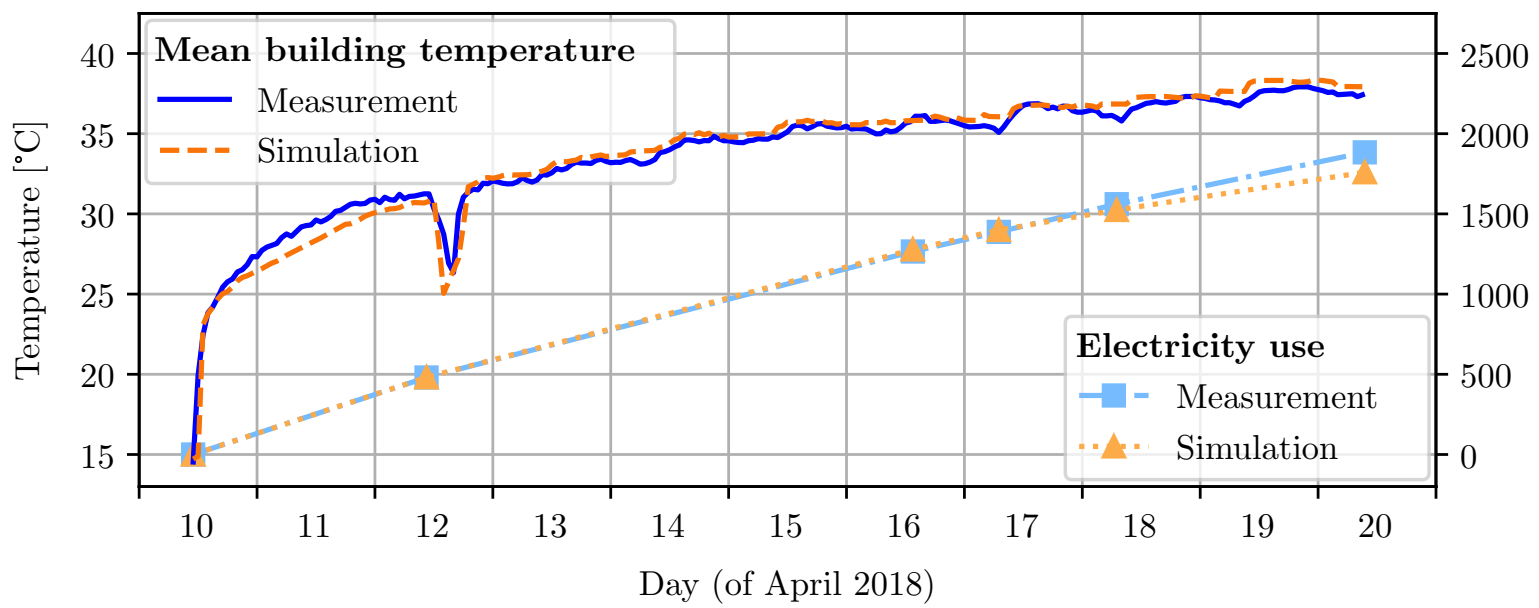

Day (of April 2018)

Figure 2: Measured and simulated mean temperature and overall electricity use for space heating during the campaign in the unoccupied building (including blower door test on April 12). The interior was heated above standard to achieve a high temperature difference to the outside environment.

Table 2: Geometry readings for different data sources and measurement methods. Inclinations are between the outside wall surface and the ground.

\begin{tabular}{|c|c|c|c|c|c|c|}
\hline Feature & Plan & $\begin{array}{c}\text { Manual } \\
\text { measurement }\end{array}$ & $\begin{array}{c}\text { Laser scan } \\
\text { point cloud }\end{array}$ & $\begin{array}{c}\mathbf{1}^{\text {st }} \text { RGB } \\
\text { point cloud }\end{array}$ & $\begin{array}{c}\text { City- } \\
\text { GML }\end{array}$ & $\begin{array}{c}2^{\text {nd }} \text { RGB } \\
\text { point cloud }\end{array}$ \\
\hline Length west wall & $9.520 \mathrm{~m}$ & $9.480 \mathrm{~m}$ & $9.480 \mathrm{~m}$ & $8.86 \mathrm{~m}$ & $8.812 \mathrm{~m}$ & $9.456 \mathrm{~m}$ \\
Length south wall & $8.545 \mathrm{~m}$ & $8.535 \mathrm{~m}$ & $8.530 \mathrm{~m}$ & $7.98 \mathrm{~m}$ & $8.263 \mathrm{~m}$ & $8.506 \mathrm{~m}$ \\
Length north wall & $8.545 \mathrm{~m}$ & $8.545 \mathrm{~m}$ & $8.542 \mathrm{~m}$ & $8.00 \mathrm{~m}$ & $8.263 \mathrm{~m}$ & $8.510 \mathrm{~m}$ \\
Building height & $9.300 \mathrm{~m}$ & - & $9.132 \mathrm{~m}$ & $8.93 \mathrm{~m}$ & $8.579 \mathrm{~m}$ & $9.132 \mathrm{~m}$ \\
Inclination north wall & $90^{\circ}$ & - & $90.27^{\circ}$ & $92.04^{\circ}$ & $90^{\circ}$ & $90.35^{\circ}$ \\
Inclination south wall & $90^{\circ}$ & - & $89.02^{\circ}$ & $88.45^{\circ}$ & $90^{\circ}$ & $89.94^{\circ}$ \\
\hline
\end{tabular}

ture profile matches the measured values, the EnergyPlus model can be considered successfully validated, and its parameters can serve as a reference for the remote sensing approach.

Regarding time, the outdoor laser scans took two hours. Another four hours were needed to align the individual point clouds. Also, augmenting the digital building model derived from the point cloud with further information required several hours of work. These tasks cannot be fully automated yet using commercially available software.

\section{Geometry reconstruction}

Table 2 compares the measured building dimensions. While the laser scan point cloud dimensions match the manually obtained values almost exactly, the first point cloud produced by Agisoft PhotoScan compresses the building in all directions due to image orientation residuals. These errors get propagated to the CityGML representation. To stabilize the point cloud, the horizontal dimensions from the terrestrial laser scans were introduced into the generation process of a second point cloud, resulting in an accuracy improvement. To avoid additional measurements, incorporating automatically logged differential GPS (DGPS) or real-time kinematics (RTK) data from the UAV are a favorable future solution to this problem. Figure 3 shows a comparison between the CityGML representation of the building and the point cloud it was computed from. Negative distance values indi-

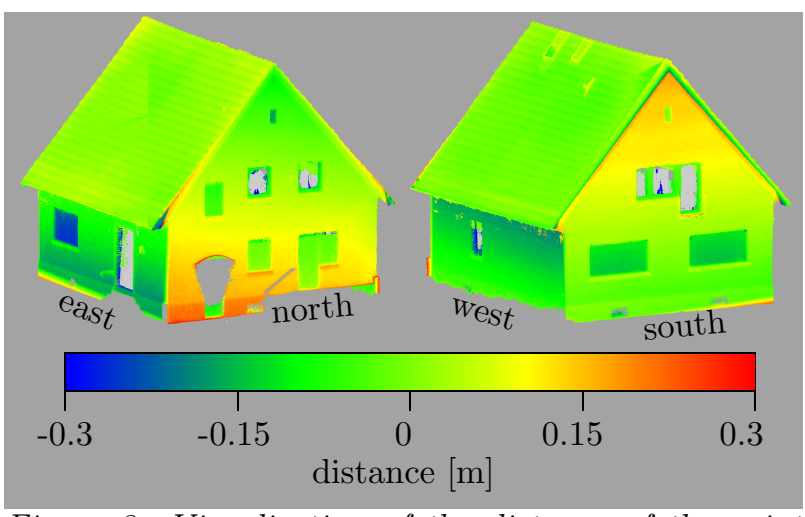

Figure 3: Visualization of the distance of the point cloud vertices to the respective CityGML surfaces (positive values for vertices outside of the hull).

cate points behind the reconstructed polygons. The inclination of the walls is orthogonal in CityGML due to algorithmic construction. The nonplanarity of the roof was successfully rectified. However, the side wall polygons lie slightly outside the point cloud, probably due to roof overhang points considered a part of the walls. All in all, almost $90 \%$ of the points are within a $5 \mathrm{~cm}$ distance to the CityGML polygons.

The identified windows are highlighted in the texture atlas in Figure 4. Eleven out of twelve main windows ( $\sim 92 \%$ ), including those with closed blinds, were detected, resulting in a total window area of $14.23 \mathrm{~m}^{2}$. There were no false positives. Only the kitchen window was not found due to a distortion of the his- 


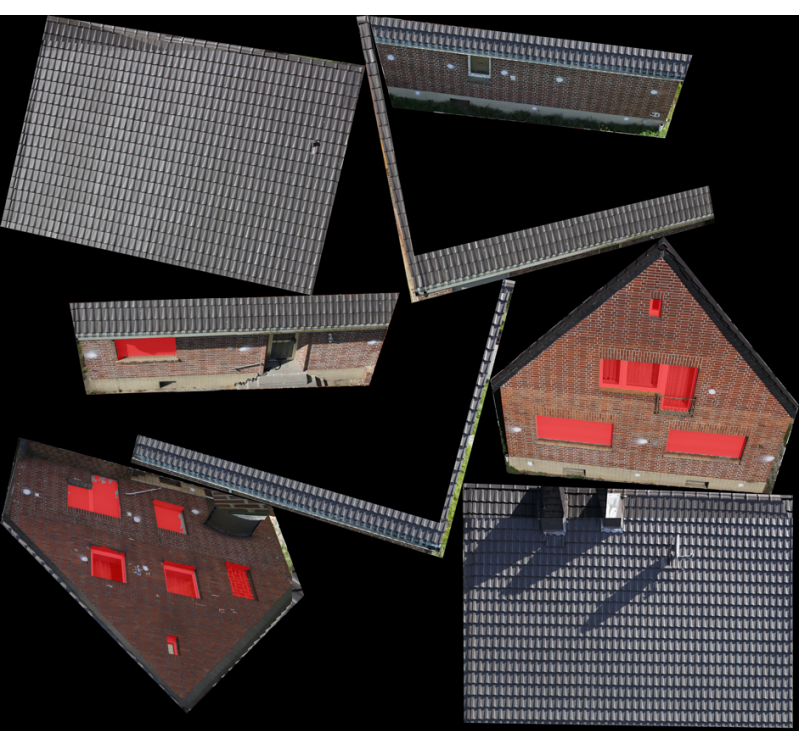

Figure 4: Envelope surface textures with detected windows marked red.

togram caused by white markers on the wall and an occluding roof overhang. Moreover, visibility issues prevented the basement windows and the side door from being identified. The front door pixels were discarded during hue channel histogram analysis because their brightness values were too different from those of the window pixels on the same wall.

Regarding the time needed for data acquisition, the nadir-looking and oblique images taken from the positions above the rooftop (see Figure 1) were recorded during a pre-planned and automatically conducted flight finished within a few minutes. To capture even small details of the façades, close-range images with a nearly horizontal orientation were taken by manually operating the UAV in a distance of $5 \mathrm{~m}$ to the building at different altitudes. As this task is not automatable yet, it consumed about 1.5 hours of time. For the generation of the point cloud, one hour for manual image selection and five to six hours for the actual calculation on a PC with an above-mainstream GPU were spent.

The number of points in the point cloud was reduced before geometrical reconstruction to lower the computation time requirements. By considering only 1.2 million instead of 12 million vertices, a decrease from more than 24 hours to 25 minutes was achieved. Subsequent wall surface texturing took six hours with four parallel processes, including the time-consuming process of stitching together multiple images to seamlessly color the façade polygons.

\section{Thermal properties of the envelope}

The U-value derived from TIR imagery was evaluated for a small region on the northern façade of the building where a heat flux meter was attached from the inside. The spot is marked as A in Figure 5. The figure also shows that radiation intensities vary among different façade regions, mostly due to different wall materials. While the HFM delivered a U-

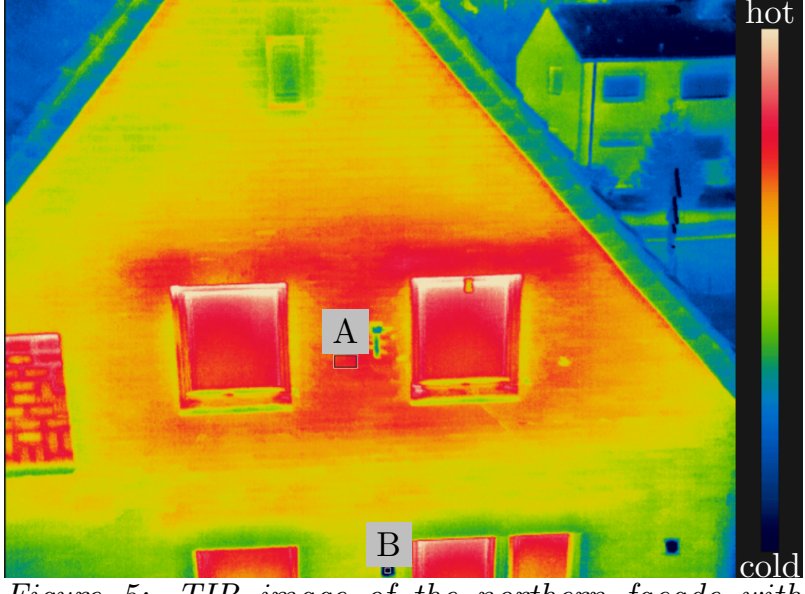

Figure 5: TIR image of the northern façade with marked areas for U-value measurement (A) and for reflected radiation measurement with crumpled aluminum foil (B).

value of $1.19 \mathrm{~W} /\left(\mathrm{m}^{2} \mathrm{~K}\right)$, the significantly higher result from quantitative TIR data was $3.5 \mathrm{~W} /\left(\mathrm{m}^{2} \mathrm{~K}\right)$. Regarding the measurement effort, no additional time is needed for TIR image recording. If the time frame just before sunrise is chosen, both TIR and RGB data can be acquired concurrently by two distinct sensors mounted to the same carrier. For the current experiment however, two separate flights were conducted.

\section{Simulation input based on remote sensing}

The height values for each upper floor were detected manually from the previously identified window polygons as depicted in Figure 6. This information was

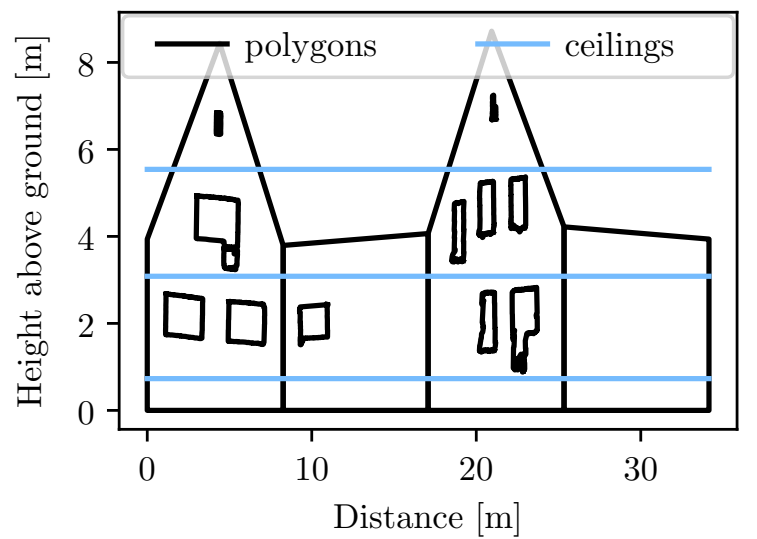

Figure 6: Projected CityGML wall polygons with ceiling heights derived from lower window edges.

introduced into the building model together with the room height of the basement $(2.07 \mathrm{~m})$. Figure 7 shows a visualization of the respective Energy ADEenhanced CityGML file.

Table 3 compares building energy simulation input parameters derived from the presented remote sensing approach with those measured conventionally (see Table 2 for the general envelope geometry). For every wall, the recognized window proportion per façade is lower for remote sensing although detection inside the images itself was accurate (see Figure 4). Apart 


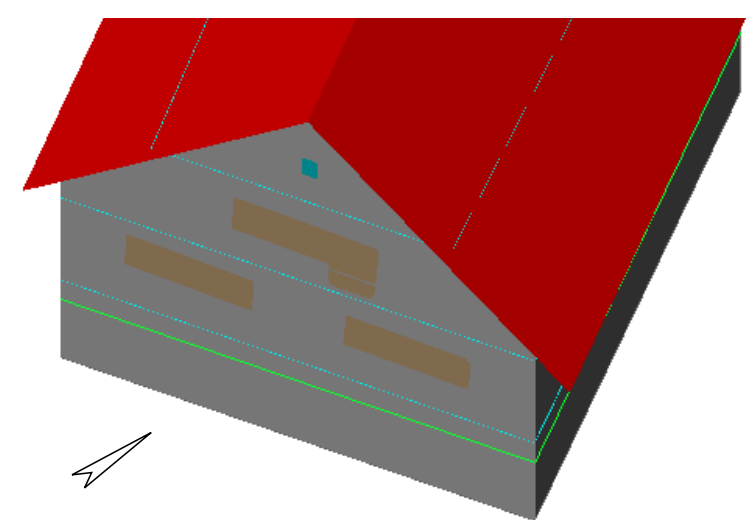

Figure 7: CityGML/Energy ADE model visualized by FZKViewer with terrain intersection (green), thermal zone borders (blue) and thermal openings (brown).

Table 3: Comparison of building energy simulation input parameters measured with conventional methods and derived from remote sensing $(R S)$.

\begin{tabular}{|cc|c|c|c|}
\hline \multicolumn{2}{|c|}{ Parameter } & Conv. & RS & Diff. \\
\hline Window & $\mathrm{N}$ & 17.5 & 11.1 & $-37 \%$ \\
area & $\mathrm{E}$ & 6.3 & 3.6 & $-43 \%$ \\
per wall & $\mathrm{S}$ & 18.8 & 14.0 & $-26 \%$ \\
{$[\%]$} & $\mathrm{W}$ & 3.0 & 0 & $-100 \%$ \\
\multicolumn{2}{|c|}{$U_{\text {wall,N }}\left[\mathrm{W} /\left(\mathrm{m}^{2} \mathrm{~K}\right)\right]$} & 1.19 & 3.5 & $+194 \%$ \\
\hline
\end{tabular}

from windows not being recognized at all (see Geometry reconstruction subsection for details), this is due to some of the detected windows being too small because of occlusions of the openings by nearby structure elements and geometric inaccuracies during window reintegration induced by image orientation errors.

\section{Discussion}

\section{Accuracy}

Results show that the general geometry of the sample building which is essential for high-level energetic simulation can be reconstructed based on photogrammetry. The derived CityGML representation slightly deviates from the physical structure in its dimensions. These issues very likely can be eliminated by incorporating advanced GPS receivers and inertial navigation systems (INS) to provide accurate initial image orientation values. This already is the case in commercial aerial photography using larger manned aircraft. In contrast to terrestrial laser scans, UAVbased imagery has the important benefit that it can comprehensively capture roof installations and features above balconies.

Regarding the detection count and area covered, window recognition on the façade images was accurate. However, the developed approach has to be tested for its applicability to other buildings with different surface materials. Nevertheless, first tests show that the exploitation of geometric features improves classification robustness even though they degrade the effective resolution of the texture atlas due to resampling in a mandatory prior rectification step. Additionally, horizontal or even up-looking images may enhance window recognition and texture mapping. To avoid picturing the rotors on these images, modifications to the UAV or image cropping are required.

U-value calculation based on TIR imagery delivered a result three times as high as the conventional measurement. This indicates that the theoretical model used is insufficient. It may be enhanced by taking the dynamic thermal behavior of the wall and atmospheric absorption of the radiation into account. Furthermore, improved calibration may reduce the uncertainty in the TIR camera radiation readings. Finally, the accuracy of the reflected radiation measured via crumpled aluminum foil has to be investigated.

\section{Time and effort}

Compared to the conventional analysis, the photogrammetric approach reduces the time for the measurements and processing from roughly a day to 2.5 hours (plus calculation time). While building reconstruction is partially hardware-accelerated, the time consumption of the visibility test and the rendering stage of the texture mapper may be improved by efficient parallel scheduling. On the other hand, since remote sensing approaches for important parameters like air infiltration rate and thermal properties of the roof have not been considered yet, their implementation is expected to cause a slight increase regarding the processing time.

\section{Model generation and data accessibility}

As it is close to the syntax used for the internal building model for data processing, CityGML and its Energy ADE is the preliminary interface to simulation tools. Although only a subset of this modelling language is supported in current simulation environments, more widespread use is expected given the number of participants in the evolution of the format (Agugiaro et al. (2018)).

As models can also be saved directly using the pickle module in Python, the high-level programming language can also be used to conveniently implement new data interfaces for buildings already processed. This includes exports to EnergyPlus input data files (idf) or to gbXML, the connection to the Modelica library AixLib (Müller et al. (2016)), or the simplification of building information for use in urban-scale simulation or in a tool like BuildingOPT (Schütz et al. (2017)) to find optimal retrofit solutions.

\section{Conclusion}

In the presented work, remote sensing and conventional measurement methods were applied for the energetic building analysis of a single-family house. Using conventional methods, a reference model was created and the necessary effort was monitored. With remote sensing, the envelope geometry and the Uvalue of a wall was measured. An approach to process the measured data into CityGML files using Energy 
ADE via an object-oriented model in Python was introduced.

A point cloud of the envelope was successfully derived from UAV-based imagery. Although the result was not noisy, it delivered smaller dimensions than the reference laser scan when generated with GPS/INS tags only. The point cloud was processed into a textured CityGML representation. About $92 \%$ of the main windows were recognized with no false positives, enabling the detection of internal ceiling positions. U-value measurement based on quantitative thermal infrared data from the outside has not delivered acceptable outcomes yet. Measurement time and manual effort were significantly reduced compared to conventional methods.

As a bottom line, it can be stated that remote sensing is able to substantially decrease effort and time needed for the generation of energy simulation models of existing buildings if the mentioned inaccuracies are overcome. Future work will also focus on the development of additional remote sensing technologies for wall material analysis and air leakage detection.

\section{Acknowledgements}

The authors would like to thank Dennis Dahlke (DLR Institute of Optical Sensor Systems) for roof overhang estimation and window detection, Martin Israel (DLR Remote Sensing Technology Institute) for his contribution regarding UAV flights and image acquisition, and RWE AG for providing access to the building in Morschenich that now serves as a reference building. The authors gratefully acknowledge the German Ministry for Economic Affairs and Energy for funding this work in the framework of the "building tomograph" (Gtom) project (03ET1405A).

\section{References}

Agugiaro, G., J. Benner, P. Cipriano, and R. Nouvel (2018). The Energy Application Domain Extension for CityGML: Enhancing interoperability for urban energy simulations. Open Geospatial Data, Software and Standards 3(1), 2.

Estevam Schmiedt, J., D. Cerra, D. Dahlke, S. Dill, N. Ge, J. Göttsche, A. Haas, U. Heiden, M. Israel, F. Kurz, M. Linkiewicz, D. Patel, M. Peichl, S. Plattner, S. Pless, B. Schiricke, C. Schorn, A. Tiddens, and X. X. Zhu (2017). Remote sensing techniques for building models and energy performance studies of buildings. In $E B C$ Annex 71: Building energy performance assessment based on in-situ measurements, Loughborough, UK.

Fischler, M. A. and R. C. Bolles (1981). Random sample consensus: A paradigm for model fitting with applications to image analysis and automated cartography. Communications of the ACM 24(6), 381-395.

Fokaides, P. A. and S. A. Kalogirou (2011). Ap- plication of infrared thermography for the determination of the overall heat transfer coefficient (U-Value) in building envelopes. Applied Energy 88(12), 4358-4365.

Frommholz, D., M. Linkiewicz, H. Meissner, and D. Dahlke (2017). Reconstructing Buildings with Discontinuities and Roof Overhangs from Oblique Aerial Imagery. ISPRS - International Archives of the Photogrammetry, Remote Sensing and Spatial Information Sciences XLII-1/W1, 465-471.

International Organisation for Standardisation (2014). Thermal insulation - Building elements - In-situ measurement of thermal resistance and thermal transmittance - Part 1: Heat flow meter method (ISO 9869-1).

Liu, Y. and D. J. Harris (2007). Full-scale measurements of convective coefficient on external surface of a low-rise building in sheltered conditions. Building and Environment 42(7), 2718-2736.

Mayer, S. (2004). Automatisierte Objekterkennung zur Interpretation hochauflösender Bilddaten in der Erdfernerkundung. Ph. D. thesis, Humboldt-Universität zu Berlin, MathematischNaturwissenschaftliche Fakultät II.

Müller, D., M. Lauster, A. Constantin, M. Fuchs, and P. Remmen (2016). AixLib - An Open-Source Modelica Library within the IEA-EBC Annex 60 Framework. In BauSIM 2016. Dresden (Germany), 14-16 September 2016.

Open Geospatial Consortium (2012). OGC City Geography Markup Language (CityGML) Encoding Standard (OGC 12-019).

Patel, D., J. Estevam Schmiedt, M. Röger, and B. Hoffschmidt (2018). Approach for external measurements of the heat transfer coefficient (U-value) of building envelope components using UAV based infrared thermography. In 14th Quantitative InfraRed Thermography Conference (QIRT). Berlin (Germany), 25-29 June 2018.

Schütz, T., L. Schiffer, H. Harb, M. Fuchs, and D. Müller (2017). Optimal design of energy conversion units and envelopes for residential building retrofits using a comprehensive MILP model. Applied Energy 185, 1-15.

Tejedor, B., M. Casals, M. Gangolells, and X. Roca (2017). Quantitative internal infrared thermography for determining in-situ thermal behaviour of façades. Energy and Buildings 151, 187-197.

Zundel, S. and I. Stieß (2011). Beyond Profitability of Energy-Saving Measures-Attitudes Towards Energy Saving. Journal of Consumer Policy 34(1), 91-105. 